\title{
La parola rivoluzionaria abbassata al corporeo: Parodia carnevalesca e trauma in Sebastiano Vassalli, Abitare il vento
}

\begin{abstract}
(italian)
Il romanzo Abitare il vento (1980), di Sebastiano Vassalli, costituisce un'interessante testimonianza della tematica dei cosiddetti "anni di piombo" nella letteratura italiana a partire dagli anni Settanta. Il protagonista, Cris, è uno sbandato uscito di galera che ha intrattenuto e intrattiene rapporti non ben definiti con la lotta armata. Attraverso un soliloquio che impasta differenti codici e registri linguistici senza soluzione di continuità, egli trascina il lettore in un'odissea tragicomica: alla ricerca di vecchie e nuove amanti, si troverà alla fine ad essere coinvolto come carceriere in un rapimento operato da una cellula di brigatisti.

Una caratteristica peculiare dell'opera è il tono comico con cui questa tematica viene affrontata; l'articolo propone dunque un'analisi della comicità del romanzo a partire dalla categoria del "carnevalesco" teorizzata dallo studioso russo Michail Bachtin. In effetti nel testo ricorrono numerosi elementi tratti dal repertorio comico scandagliato dal critico nel suo studio dell'opera di Rabelais: tra questi risalta l'abbassamento declassante del lessico rivoluzionario alla sfera semantica del basso materiale e corporeo.
\end{abstract}


Individuati gli elementi carnevaleschi del romanzo, l'articolo si interroga sulle funzioni di questa comicità, che si dispiega come critica parodica dell'ideologia, ma anche come strategia di "derealizzazione" finalizzata ad allontanare un'esperienza storica vissuta come trauma.

\section{Abstract (english)}

Sebastiano Vassalli's novel Abitare il Vento (1980) is an interesting testimony to the theme of the so-called "anni di piombo" in Italian literature since the Seventies. The protagonist, Cris, just released from jail, has had and still has ambiguous relationships with the armed struggle. Through a soliloquy that mixes different linguistic codes and registers, he drags the reader into a tragicomic odyssey: in search of old and new lovers, he will ultimately be involved as a jailer in a kidnapping operated by extreme left terrorists.

A peculiar feature of the work is the comic tone with which this subject is treated; the article therefore proposes an analysis of the novel's comedy from the category of "Carnival" theorized by Michail Bachtin. Indeed, in the text, there are many elements from the comical repertoire that the critic defined in his study of the work of Rabelais, among which the declassing lowering of the revolutionary lexicon to the semantic sphere of the material and the body. 
Once the carnival elements of the novel have been identified, the article explores the functions of this comedy: a parody of ideology, but also a "derealization" strategy aimed at removing a historical experience lived as a trauma.

\section{Keywords}

Sebastiano Vassalli, Michail Bachtin, Abitare il vento, comico, anni di piombo

\section{Abitare il vento}

Nel 1980 per i tipi di Einaudi esce il romanzo Abitare il vento, di Sebastiano Vassalli. Alle soglie del decennio che chiuderà definitivamente la stagione delle proteste e delle rivolte, la delusione dell'autore per l'esperienza letteraria e politica inaugurata con l'adesione al Gruppo 63 (a partire almeno dal convegno di Fano del 1967), si è completamente consumata nelle opere a cavallo tra gli anni Settanta e Ottanta. Vassalli, preludendo al periodo del cosiddetto "riflusso", mette dunque in scena nel libro il naufragio dei miti che avevano plasmato quelle stesse lotte e contestazioni, scegliendo di utilizzare il linguaggio della farsa piuttosto che quello della tragedia.

Il romanzo consta del lungo flusso di coscienza di Antonio Cristiano Rigotti detto Cris: un giovane sbandato appena uscito di galera, che ha intrattenuto e intrattiene rapporti non ben definiti con la lotta armata. Attraverso un soliloquio che impasta differenti codici e registri linguistici senza soluzione di continuità, Cris trascina il lettore in un'odissea 
tragicomica: alla ricerca di vecchie e nuove amanti su e giù per la penisola, si trova ad essere coinvolto come carceriere in un rapimento operato da una cellula di terroristi di estrema sinistra. Allontanato in quanto sospettato di essersi fatto individuare dalla polizia, viene tradito dai presunti amici e, impossibilitato dunque a fuggire, decide di impiccarsi. Ma prima di compiere il gesto estremo, con un ultimo atto metafinzionale, si rivolge direttamente a chi ha scritto la sua storia, chiedendo di conferire con l'autore del romanzo «inchiodato finalmente al trave delle sue responsabilità» (Vassalli, 2008: p.106).

Quelli che il protagonista incontra in una vera e propria discesa agli inferi sono «fantasmi degli anni di piombo, né eroici, né iperrazionali: solo farseschi» (Nesi, 2005: p.46), i quali lo trascinano in un mondo dal quale tenta, continuamente e invano, di emanciparsi. Come ha notato Cristina Nesi (2005), Cris «racconta la sua vita balorda ed errabonda, quella di un giovane che vorrebbe vivere fuori dal tempo e dalle ideologie, nel vento appunto, e finisce invece per essere sempre più invischiato nel proprio tempo e, seppur casualmente, nella lotta armata» (p.46). Le sue erranze dunque non sono che una vana fuga dall'estremismo politico dei cosiddetti "anni di piombo", cui potrà sottrarlo solo la morte con la quale si conclude il romanzo.

Il fallimento delle ideologie e la demitizzazione del Sessantotto costituiscono del resto il tema chiave delle opere di Vassalli a cavallo tra anni Settanta e Ottanta; tema che sarà 
successivamente ripreso in Archeologia del presente (2001). Abitare il vento si inserisce infatti tra altri due romanzi in cui il tono comico dominante è volto alla demistificazione parodica: L'arrivo della lozione (che però sposta la prospettiva negli ambienti neofascisti, 1976) e Mareblù (1982). Nel primo troviamo la ricostruzione agiografica della parabola tragicomica di un delinquente del sud Italia che diventa terrorista di estrema destra; nel secondo il protagonista è il guardiano di un camping sulla riviera ligure, un alienato che combatte la sua personalissima lotta di classe contro i villeggianti borghesi, intrattenendo un fitto dialogo con i ritratti di Marx, Mao, Lenin e Stalin appesi nella sua stanza. Si viene a costituire dunque, all'interno della bibliografia dell'autore, una sorta di trilogia comica che prende di mira i miti cardine della sua generazione. Tra questi, soprattutto il mito della "Rivoluzione", scempiata in "Luzione" e mutata in "Lozione" nel testo del 1976, e seppellita poi definitivamente dalla risata del protagonista con cui si chiude Mareblù: «Io Augusto Ricci per la prima volta dopo sessant'anni di vita seria finalmente rido di gusto, sghignazzo: ah ah ah ah ah ah ah ah ah.[...] Rido come un pazzo, ah ah.» (Vassalli, 1982: p.190).

È difatti la comicità, declinata in forme distinte, che funge da trait d'union di queste tre opere pur molto diverse tra loro. Ma di che genere di comico si tratta? E che funzione svolge in rapporto alla materia trattata? La critica contemporanea, nello specifico Maria 
Corti (1978), ha riconosciuto nelle opere di Vassalli degli anni Settanta una «linea satirica sottesa già ai disegni dei primi scritti» (p.135), individuando la «satira sociale» come «motivo ricorrente» (p.161); occorre però tenere in conto che il testo di Corti, uscito nel 1978, si riferisce a lavori precedenti Abitare il vento, quali lo pseudo-trattatello Tempo di màssacro (1970) e L'arrivo della lozione (1976).

Con il romanzo che qui prendiamo in esame Vassalli compie invece un salto di qualità per cui la terminologia legata al genere della satira (e al riso ad essa connaturato) non è più sufficiente. Ci sembra difatti che per comprendere il tono comico di Abitare il vento, rispetto agli altri libri descritti da Corti, risulti più produttiva una lettura in chiave "carnevalesca", sul modello di quella utilizzata da Michail Bachtin nello studio dell'opera di François Rabelais (sebbene non manchino interpretazioni satiriche anche di questo romanzo, come quella avanzata da Giovanna Ioli, 1982). La distinzione che qui proponiamo discende da quella operata dal critico russo tra riso satirico e riso carnevalesco: se la satira possiede una carica esclusivamente negativa, atta a moralizzare e demolire, secondo Bachtin il comico carnevalesco trova la sua caratteristica precipua nell'ambivalenza. Il riso del carnevale infatti abbassa per innalzare, uccide per rinnovare e rigenerare, distrugge per ricostruire; la satira si limita invece alla critica negativa e alla fustigazione dei costumi, connotandosi così come comicità reazionaria per eccellenza. In 
effetti ci sembra che L'arrivo della lozione e Abitare il vento si possano entrambi inquadrare nel genere della parodia, ma il primo aderisce meglio ad un modello parodico moderno, dove si attua «una ricerca di effetti satirici tramite la variazione complessa dei registri linguistici»(Nesi, 2005: p.44), mentre il secondo attinge ad un repertorio più propriamente legato alla comicità del carnevale. La differenza tra i due paradigmi è così descritta da Bachtin (1979b): «la parodia carnevalesca è lontanissima dalla parodia moderna, che è puramente negativa e formale; la parodia carnevalesca, infatti, negando, al tempo stesso fa resuscitare e rinnova» (pp.14-15). Consideriamo, per cominciare, il rapporto tra l'autore e la propria opera: per il critico russo (1979b),

l'autore puramente satirico, che conosce soltanto il riso negativo, si pone al di fuori dell'oggetto della sua derisione, vi si contrappone, e così viene distrutta l'integrità dell'aspetto comico del mondo, e ciò che è 'comico' (negativo) diventa un fenomeno privato. (p.16)

In Abitare il vento l'autore viene invece tratto nel gioco parodico dallo stesso protagonista, che lo inchioda, come abbiamo visto, «al trave delle sue responsabilità»: si apre una dimensione metaletteraria per cui il personaggio è cosciente della sua qualità finzionale, e allo scrittore viene così permesso di affacciarsi nel testo da lui prodotto, trascinato in tal 
modo nel vortice della parodia. Quella che si propone di seguito è dunque una lettura del romanzo di Vassalli attraverso l'ottica della “carnevalizzazione” così com'è stata definita dal critico russo. La lettura carnevalesca del comico adottato dall'autore in Abitare il vento è utile per comprendere il rapporto tra Vassalli e la materia trattata, ossia gli "anni di piombo": qual è la funzione di questa comicità? Vedremo come la demitizzazione delle grandi ideologie degli anni Settanta si accompagna a strategie di straniamento proprie della rielaborazione di un'esperienza traumatica.

\section{Gli elementi carnevaleschi nel romanzo}

In effetti, in Abitare il vento colpisce la presenza di numerosi elementi tratti dal repertorio comico del carnevalesco scandagliato da Bachtin, a partire dalla caratterizzazione del personaggio principale. Il protagonista si presenta infatti come «Antonio Cristiano Rigotti detto Cris, di professione cavaliere errante, amico di tanti e di tante» (Vassalli 2008: pp.11-12), e questa dicitura ritornerà, quasi un epiteto fisso, in numerosi passi del romanzo. «Cavaliere errante» è anche lo pseudonimo con cui egli firma i giochi enigmistici di cui è prolifico inventore, e che nel testo si intrecciano ad intermittenza con il discorso principale. Questa figura letteraria così come risulta deformata dal meccanismo parodico (poiché Cris altro non è che un personaggio allo sbando), inoltre, 
rimanda immediatamente a Don Chisciotte, parodia carnevalesca per eccellenza. Cris dichiara in numerosi passi del testo che la sua nobile aspirazione (il fine della sua quête, potremmo dire) è «abitare il vento», ma in realtà il suo errare è diretto unicamente dalle pulsioni sessuali, incarnate dal fallo che egli personifica con tono osceno e dissacrante tramite il soprannome di «Grande Proletario». Dapprima dunque dal Lazio, dove è appena uscito di galera, egli si reca a Firenze, sulle tracce di una sua vecchia fiamma; ma è costretto a deviare le proprie pulsioni su altre due ragazze, e qui viene coinvolto incidentalmente nei traffici della lotta armata. Svincolatosi da questa prima cellula terroristica, riesce a raggiungere Milano, ma si ripete lo stesso identico copione: ancora una volta il soddisfacimento degli istinti sessuali si intreccia al coinvolgimento nel terrorismo, ed è allora che Cris si ritrova a fare da carceriere ad un giovane ostaggio per conto di un gruppuscolo di brigatisti. Il protagonista risale l'Italia in cerca di vecchi e nuovi amori, ma il suo errare non lo condurrà se non al suicidio: in questo senso la quête parodica si configura come una vera e propria catabasi. Il movimento verso il basso è d'altronde descritto da Bachtin (1979b) come direzione fondamentale della comicità carnevalesca:

l'orientamento verso il basso è proprio di tutte le forme di allegria festiva e popolare e del realismo grottesco. In basso, alla rovescia, all'incontrario: tale è il movimento di tutte queste 
forme, che fanno precipitare tutto verso il basso [...] sia sul piano dello spazio reale che su quello metaforico. (p.407)

In particolare, all'interno del repertorio di situazioni comiche carnevalesche, «l'orientamento verso il basso è proprio delle baruffe, delle lotte e delle botte» (Bachtin, 1979b: p.407). Vediamo dunque che anche questa componente non manca nel romanzo, precisamente nell'episodio in cui Cris picchia un prete che è venuto a cercarlo a casa per redimerlo. Il passo è tanto più significativo in quanto, essendo la vittima delle botte un rappresentante di un'istituzione dominante quale la Chiesa, descrive una vera e propria scena di detronizzazione carnevalesca, durante la quale il re viene ritualmente sbeffeggiato e malmenato. «Allora», racconta il protagonista, «gli branco il collo che con una mano sola non ce la faccio e mi tocca usarle tutt'e due e lo inchiodo sopra la rete che lui subito distende le braccia in croce come il suo antenato, il Famoso, e scagazza sangue da naso» (Vassalli, 2008: p.36). Il riferimento cristologico («il suo antenato, il Famoso») fa emergere l'elemento parodico e allo stesso tempo l'ambivalenza delle botte carnevalesche, che «rovesciano, gettano a terra, calpestano. Seppelliscono. Ma nello stesso tempo sono creatrici: gettano i semi e raccolgono i frutti» (Bachtin 1979b: p. 407).

La funzione rigeneratrice di questo riso è inoltre esplicitata da Cris nel momento in cui definisce la propria filosofia di vita, nell'ottica di una concezione ciclica del tempo propria 
della cultura popolare: «il futuro solo pensato è una lastra di vetro che riflette il passato e poi anche del futuro a me non frega gnente, io mi costringo a pensare al presente. Secondo la regola prima e universale del cavaliere errante che tutto è tondo che tutto è gioco e che tutto fortunatamente dura poco» (Vassalli, 2008: pp.46-47). Da queste ultime parole traspare una visione del mondo in tutto e per tutto carnevalesca, dove ogni cosa è relativizzata in un gioco di avvicendamenti, mutamenti e continui rovesciamenti.

Tenendo poi ben presente che «tutti questi abbassamenti non hanno un carattere relativo né astrattamente morale, ma $[\ldots]$ tendono verso un centro assoluto e positivo, verso il principio della terra e del corpo, che assorbe e dà la vita» (Bachtin, 1979b: p.407), per un'interpretazione carnevalesca del romanzo di Vassalli risulta ineludibile la ricognizione delle immagini legate al corpo nelle sue funzioni più "basse". Prendiamo ad esempio la figura, potenzialmente tragica, dell'ostaggio: essa viene tratteggiata a partire da un'oggettivazione (viene chiamato «il merce») e la sua identità viene ridotta ad uno spropositato espletamento delle funzioni corporali più oscene e dunque profondamente legate alla comicità carnevalesca:

il merce $[\ldots]$ attacca coi vomiti, vomita a più non posso con la testa dentro nel secchio, scaracchia e qui le cose si fanno serie [...] poi di colpo attacca a cacare e inzomma prima che 
c'infilo il secchio si sporca attorno parecchio, o. Forse è la volta che si svuota, piscia dal culo e peta e tira puzze bestiali [...]. (Vassalli, 2008: p.50)

La riduzione dell'ostaggio alla dimensione materiale-corporea è sancita definitivamente dalla sua identificazione tramite un soprannome che rimanda esclusivamente agli escrementi: il ragazzo «tanto per cambiare si chiama Andrea, tutti i ragazzi di diciassette-diciotto anni oggi si chiamano Andrea, ma io lo chiamo Diarrea perché ci ho le mie ragioni» (Vassalli, 2008: p.55), afferma Cris. Il trattamento iperbolico del piano materiale-corporeo dà vita a quella che Bachtin (1979a) definisce «eroicizzazione rabelesiana di tutte le funzioni della vita corporea: del mangiare, del bere, del defecare, del coire»; il processo di «iperbolicizzazione» di tali atti «li priva della loro banalità, del loro colorito usuale e naturalistico» (p.340). Anche Erich Auerbach (1972), nel capitolo di Mimesis intitolato Il mondo nella bocca di Pantagruele riconosceva questa «eroicizzazione» delle funzioni corporali, osservando come la «visione creaturale dell'uomo» tipica del Medioevo venisse ribaltata di segno: non più memento mori ma vitalità esasperata. In Rabelais, dunque, questa visione

non ha più, come nel realismo della fine del Medioevo, un tono fondamentale di lamento sulla caducità del corpo e di tutto ciò che è terreno; il realismo creaturale ha in Rabelais un 
significato nuovo, nettamente opposto a quello medievale, quello di trionfo vitalisticodinamico dell'essere corporeo e delle sue funzioni. (p.18)

Nel romanzo di Vassalli è invece la carica mortifera delle ideologie che viene presa di mira dalla demistificazione; e precisamente nel trattamento iperbolico delle immagini che afferiscono al piano materiale-corporeo risiede l'afflato vitalistico con cui il giovane Cris, nell'affermazione esasperata della propria corporeità, tenta invano di sottrarvisi. La dimensione concreta del corpo fa da contrappunto all'astrattezza dell'ideologia.

Difatti, tra queste funzioni della vita corporea, è l'elemento sessuale che risulta significativamente preponderante nel romanzo di Vassalli: il fallo del protagonista (che, come abbiamo detto, incarna le pulsioni sessuali, unico reale motore delle sue erranze) viene addirittura personificato, diventando un vero e proprio personaggio della storia con cui Cris intrattiene dibattiti e discussioni. Il riferimento al fallo è d'altronde tipico del carnevalesco: in particolare, secondo Bachtin (1979b), tra le immagini corporee di cui si nutre il realismo grottesco, «l'accento è messo su quelle parti del corpo in cui esso è aperto al mondo esterno, in cui cioè il mondo penetra nel corpo o ne sporge, oppure in cui il corpo sporge sul mondo» (p.32).

In questa preponderanza della tematica sessuale c'è forse un richiamo, tra le altre fonti, al Lamento di Portnoy di Philip Roth, pubblicato in traduzione da Bompiani nel 1969 e che 
Vassalli potrebbe quindi aver letto prima di comporre Abitare il vento. Il testo, che si dipana come una lunga confessione lamentosa del giovane ebreo americano Aleander Portnoy al suo psicanalista, dottor Spielvogel, potrebbe in effetti aver intercettato gli interessi del giovane Vassalli, il quale si laureò in Lettere a Milano discutendo con Cesare Musatti una tesi su "La psicanalisi e l'arte contemporanea" (Vassalli and Tesio, 2010: pp.2425).

Anche nell'opera di Roth il monologo del protagonista sottintende una visione ludica del mondo, pur abbozzata e meno chiaramente bachtiniana di quella di Cris, in particolare nel paragone tra la vita e una partita di baseball (Roth, 2014: p.195) e nel frequente inquadramento delle vicende del protagonista nel repertorio dell'umorismo ebraico: «io sono il figlio in una barzelletta ebraica... solo che non è affatto una barzelletta!» (Roth, 2014: p.32). Quello che più colpisce nel confronto tra i due romanzi è però, come accennato, il comune trattamento della tematica sessuale: nei diversi capitoli dedicati al rapporto tra il protagonista monologante e il sesso, anche l'ansia di Portnoy, come quella di Cris, viene presentata infatti come una quête mossa da un desiderio continuamente instabile e inappagato: «tette e fighe e gambe e labbra e bocche e lingue e buchi del culo! Come posso rinunciare alla novità, visto che una ragazza, per quanto deliziosa e provocante sia 
stata un tempo, mi diventerà inevitabilmente familiare come un pezzo di pane?», chiede infatti la voce narrante allo psicanalista (Roth, 2014: p.85).

Ma, tornando a Vassalli, ai fini della nostra analisi risulta molto interessante nello specifico l'epiteto con cui l'organo genitale viene personificato. Accade infatti che nelle prime pagine del romanzo Cris, che ha caricato in macchina due giovani autostoppiste tedesche, descrive così la sua eccitazione alla vista delle gambe delle ragazze: «op là: il Grande Proletario si è mosso. D'istinto nel buio della brachetta. Come l'ago magnetico della bussola, verso l'oriente che è rosso» (Vassalli, 2008: p.10). Ci si può innanzitutto soffermare sul termine «brachetta»: l'indumento designato con questa parola ha un significato particolare nel Gargantua di Rabelais, in cui è spesso associata al fallo e a cui vengono conferite virtù taumaturgiche. Un termine leggermente differente da quello utilizzato in Abitare il vento, «braghetta», si trova in numerose ricorrenze sin dalla traduzione di Gildo Passini del 1925 - in cui però, in singolare occorrenza, viene utilizzato anche «brachette» (Rabelais, 2012: p.265) - e, significativamente, in quella di Mario Bonfantini per l'edizione Einaudi del 1973, che lo stesso Vassalli potrebbe aver letto. Basti qui citare il passo che descrive la vestizione del piccolo Gargantua:

per la braghetta presero sedici canne e un quarto di quello stesso panno. E la sua forma fu come quella degli archi di sostegno: assai graziosamente fermata con due belle fibbie d'oro, 
chiuse con due ganci di smalto, in ciascuno dei quali era incassato un grosso smeraldo della grandezza d'una melarancia. Perché $[. .$.$] questa pietra ha virtù erettiva e confortativa del$ membro naturale. L'apertura della braghetta era della lunghezza d'una canna [...]. (Rabelais, 1973: p.30)

Ma risulta molto interessante anche il richiamo al «Grande Proletario». Da questo punto in avanti infatti, nel libro il protagonista si riferirà sempre al proprio organo sessuale con tale formula. L'espressione mutuata dal celebre discorso di Pascoli vuole sicuramente fare il verso ad una cultura letteraria borghese nutrita dalle frasi fatte della letteratura («Ho fatto il liceo classico prima del Sessantotto, io!» rivendica in continuazione Cris), ma tende soprattutto a ridicolizzare le parole chiave della contro-cultura rivoluzionaria dell'epoca, come emerge dal riferimento all'«oriente che è rosso». Ciò si fa evidente nel momento in cui anche l'analisi di classe propria del marxismo viene tradotta in termini puramente corporei. Poche pagine dopo, alla vista dei «pancemolli romani di ministero in trasferta nel ristorante bono, sul mare», Cris commenta infatti così: «questa è la caratteristica prima del panciamolla di città e di paese, che essendo assolutamente sprovvisto di Grande Proletario lui ha il piccolo borghese» (Vassalli, 2008: p.12). Così le parole d'ordine della cultura antagonista che ha diretto le mosse di un'intera generazione vengono abbassate alla sfera semantica del corporeo, in un procedimento tipico del carnevalesco. «L'abbassamento», 
secondo Bachtin (1979b), «è il principio artistico anche del realismo grottesco: tutte le cose sacre e alte sono reinterpretate sul piano del 'basso' materiale e corporeo, o messe in correlazione e mescolate alle immagini di questo 'basso'» (p. 407).

La necessità della demistificazione di queste espressioni formulari, rigidamente stereotipate e connaturate al'ideologia, in Vassalli è strettamente legata alla percezione dell'aura di sacralità immutabile che circonda il regime discorsivo della "rivoluzione"; una percezione che l'autore ha trasposto nel recente romanzo Le due Chiese (2010) ed ha in seguito esplicitato, ad esempio, in una conversazione con Giovanni Tesio:

il socialismo, che poi ha visto la sua formulazione dottrinaria nelle opere di Karl Marx e ha generato nel mondo i vari comunismi, si basa sulla religione del lavoro [...] aveva il suo popolo eletto nella classe operaia (il 'proletariato' di Marx); celebrava i suoi riti nella festa del lavoro, negli scioperi e nelle occupazioni delle fabbriche; aveva i suoi sacerdoti nei sindacalisti e nei funzionari di partito; aveva la sua parusìa in un atto di trasformazione violenta: la rivoluzione, che attraverso la dittatura del proletariato sulle altre classi sociali doveva portare alla società perfetta. (Vassalli and Tesio, 2010: p.68)

Anche nell'opera di Roth troviamo d'altronde la personificazione del fallo e il dialogo del protagonista con il suo membro (Roth, 2014: p.102); e occorre qui menzionare come pure nel 
Lamento assuma importanza il termine con cui l'organo sessuale viene definito. Se infatti il linguaggio percepito come sacrale da Vassalli è quello della "Rivoluzione", Alex Portnoy non intende tanto demistificare un'ideologia politica, quanto liberarsi dall'eredità della cultura ebraica che sente pendere su di sé come una condanna. Così, nonostante quest'ansia di liberazione pervada le lamentele del protagonista, il suo monologo è denso di espressioni desunte dallo Yiddish; tra queste spicca, per l'appunto, il termine con cui Alex si riferisce invidioso al pene del padre nei suoi ricordi infantili:

Shlong: la parola dipinge con una certa precisione la brutalità, la carnevolezza che io tanto ammiro, il dondolio cieco, inconscio e ponderoso di quella canna vivente da cui mio padre sprizza getti d'acqua spessi e forti come una fune...mentre io emetto spaghetti giallastri che la mia eufemistica madre definisce "sis". (Roth, 2014: p.42)

Non manca però, sul finale del Lamento, anche l'abbassamento al piano materialecorporeo dello stesso linguaggio politico cui attinge Vassalli e che non è estraneo al protagonista (Portnoy si potrebbe infatti definire come un progressista radicale), anche se viene messo sempre in relazione all'emancipazione dall'eredità culturale ebraica della Diaspora: 
certo non mi era mai passato per la testa che avrei finito per liberare dalla cattività null'altro che il mio cazzo. LASCIATE LIBERO IL MIO PISELLO! Ecco lo slogan di Portnoy. Ecco la storia della mia vita, riassunta in cinque eroiche parole sconce. Una parodia! La mia politica mi è finita interamente nella nerchia! SEGAIOLI D.O.C. DI TUTTO IL MONDO UNITEVI! NON AVETE ALTRO DA PERDERE CHE IL CERVELLO! (Roth, 2014: p.201)

In questa parodia sessualizzata del Manifesto del Partito Comunista di Marx ed Engels è difficile non riconoscere un possibile precedente del «Grande Proletario» di Cris.

\section{Un linguaggio delle «idee senza parole»}

Qual è dunque il fine di questo abbassamento carnevalesco? L'utilizzo delle parolechiave del discorso antagonista per designare organi sessuali o, in ogni caso, referenti materiali o corporei che nell'accostamento svalutano le parole e le locuzioni ricorrenti nel linguaggio rivoluzionario privandole del loro significato politico, è volto a rendere patente il vuoto che si cela dietro a tali espressioni. Un'operazione di questo tipo si inquadra perfettamente nella ricerca letteraria dell'autore, e precisamente nella ricerca sul linguaggio, un interesse maturato durante l'esperienza della neoavanguardia e testimoniato

negli anni seguenti anche dal saggio Il neoitaliano. Le parole degli anni Ottanta scelte e 
raccontate da Sebastiano Vassalli (1989). Come ha infatti precisato Cristina Nesi (2005), dal contatto con il Gruppo 63 Vassalli «conserva in eredità l'interesse per i processi di omologazione del linguaggio, che traspaiono dalla parodia delle banalità linguistiche e dalla decantazione del lessico massificato»; un interesse accentrato, sin da L'arrivo della lozione, «sullo scialo delle parole vuote, nate "deliberatamente, per ingannare"» (pp.15-16). In particolare, l'autore in questi anni riflette su «quanto il gergo politico [...] rechi "in sé il massimo qualitativo di violenza simbolica e quindi di silenzio civile"»e

per esorcizzare la violenza simbolica, esercitata sul linguaggio e attraverso di esso, riflette sulle parole che attingono ad immagini mitiche deformi e nelle quali possono, come è avvenuto nel nazismo, venir proiettate certe ideologie (Karl Kerényi le avrebbe definite 'miti tecnicizzati') oppure su quelle che un tempo erano piene e che la comunicazione politica ha svuotato (come 'democrazia' o 'antifascismo'). (Nesi, 2005: pp. 44-45)

Difficile non pensare al «linguaggio delle idee senza parole» su cui rifletteva pochi anni prima (a partire proprio dal concetto di "mito tecnicizzato" di Kérenyi) il mitologo Furio Jesi (2011). Si tratta di un linguaggio che, nella definizione dello studioso torinese, 
presume di poter dire veramente, dunque dire e al tempo stesso celare nella sfera segreta del simbolo, facendo a meno delle parole, o meglio trascurando di preoccuparsi troppo di simboli modesti come le parole che non siano parole d'ordine. Da qui la disinvoltura nell'uso di stereotipi, frasi fatte, locuzioni ricorrenti. (pp.27-28)

Se le «idee senza parole» venivano individuate quale fondamento della Cultura di destra, non bisogna dimenticare come lo stesso Jesi avesse ben presente che in tale definizione era possibile inquadrare una certa retorica propria della sinistra:

vi sono buone ragioni di allarmarsi - ed è perfino ovvio dirlo - quando in numerosi discorsi celebrativi proprio della Resistenza ricompare il linguaggio delle idee senza parole. Delle “idee senza parole" è spesso anche il sinistrese, compreso quello più dinamitardo - affine in ciò al parlare dei suoi avversari istituzionali. (Jesi, 2011: pp.26-27)

Escludendo il riferimento alla Resistenza, è proprio su questo cortocircuito che agisce la “carnevalizzazione” nel romanzo di Vassalli: sulla trasformazione del linguaggio della "Rivoluzione" in un mito nel senso deteriore del termine; ovvero, potremmo dire sempre con Jesi, sulla trasformazione del regime discorsivo della "Rivoluzione" in una "macchina mitologica", in cui le espressioni ricorrenti e gli stereotipi (che verrebbero ad esserne i 
mitologemi) vanno a comporre una barriera impenetrabile che promette di celare un contenuto ("ill" mito, per l'appunto) ma che in realtà al suo interno non ha se non un vuoto. Vassalli attinge al repertorio (per lo più linguistico) di quella che percepisce, paradossalmente, come cultura dominante, ma ne cambia radicalmente il segno attraverso l'iperbolicizzazione e l'accostamento inusitato e svalutante. Stante il paragone tra socialismo e Chiesa che abbiamo ritrovato nelle parole dello stesso autore, riconosciamo nel romanzo l'abbozzo del medesimo meccanismo del dissenso che Auerbach (1972) descriveva nel tracciare la relazione tra il Gargantua e la cultura cristiana medievale della quale era profondamente innervato il repertorio di immagini a cui faceva riferimento Rabelais. L’imitazione «realistica, e superrealistica» dell'autore francese, infatti,

si volge alla trionfante vita terrena, ed è completamente anticristiana; ed è anche opposta ai sentimenti di cui ci perviene l'eco dal realismo creaturale del tardo Medioevo, tanto opposta che è proprio nei tratti medievali del suo stile che più chiaramente si rivela il suo discostarsi dal Medioevo; essi hanno mutato del tutto scopo e funzione. (pp.18-19)

Anche in Vassalli la critica della cultura dominante si compie attraverso il trattamento comico e iperbolico dei materiali peculiari di tale cultura, anche se nell'autore di Abitare il vento questi materiali sono parole, formule e stereotipi piuttosto che immagini come quelle 
proprie del realismo creaturale medievale cui attinge a piene mani Rabelais. Il procedimento è però lo stesso: mediante l'abbassamento delle parole-chiave del discorso dominante al piano materiale-corporeo, Vassalli compie la parodia e la "carnevalizzazione" della cultura alla quale mira la demitizzazione.

In questo senso Abitare il vento veicola quello che Bachtin (1979b) definiva un «discorso impavido»: utilizzando gli espedienti del carnevalesco, il comico diventa una presa di parola tesa a demistificare le verità e le parole d'ordine del discorso percepito come egemone. «Durante i millenni», afferma il critico russo, «il popolo si è valso dei diritti e delle libertà delle immagini comiche della festa per dar corpo in esse al suo profondo senso critico, alla sua profonda diffidenza nei confronti della verità ufficiale»; dunque in tale contesto si veniva a generare un discorso proprio del popolo stesso, «che si esprimeva sul mondo e sul potere senza scappatoie né reticenze» (p.295). Cris, parodia del cavaliere errante, si può pertanto inquadrare nella triplice figura del furfante/buffone/sciocco, personaggio che sfrutta una posizione privilegiata rispetto alla cultura dominante. Una posizione da cui gli è consentito vederne ed esplicitarne le storture, giacché a queste tre figure «sono intrinseci una peculiarità e un diritto: essere estranei in questo mondo. Essi infatti non solidarizzano con alcuna condizione di vita di questo 
mondo, da nessuna di esse sono soddisfatti e di tutte vedono il rovescio e la menzogna» (Bachtin, 1979a: p.306).

Il romanzo di Vassalli non attinge certo alle stesse fonti del popolare da cui sorge l'opera di Rabelais, ma assume in ogni caso questa funzione di «discorso impavido» attraverso gli espedienti della comicità carnevalesca. Il tutto tramite la lente del paradosso: il discorso che si proponeva come antagonista si è costituito a sua volta come regime discorsivo dominante e deve perciò essere decostruito e demistificato. Un medesimo procedimento di risemantizzazione della parola "sacra" avviene nel titolo dell'opera: l'espressione «abitare il vento» è infatti tratta dal libro dei Proverbi $(11,29)$, in cui possiede una connotazione negativa: «chi manda in rovina la propria casa abiterà il vento». Nel romanzo di Vassalli il segno viene invece totalmente capovolto in un'accezione positiva, e l'espressione designa quell'affrancamento dalle ideologie che Cris non riesce tuttavia a raggiungere se non mediante il suicidio.

Per concludere la rassegna degli elementi carnevaleschi di Abitare il vento, è opportuno sottolineare come anche la scena del suicidio del protagonista riveli un'attitudine profondamente ambivalente; anzi, proprio nel finale, in cui egli si impicca ostentando il fallo eretto, si riassume l'intero senso comico del romanzo. Il fallimento esistenziale di Cris 
- che è il fallimento di un'intera generazione - non assume infatti la consistenza tragica di cui necessiterebbe: come sottolinea Nesi (2005),

del Sessantotto resta ormai solo il ricordo di un vuoto e di un naufragio, in cui molti hanno scelto di scomparire, consapevoli che "intorno a loro non c'è più niente in cui $[\ldots]$ credere". Ma chi soccombe, non sa dalle sue disgrazie innescare la tragedia. Tutt’al più la farsa, con il lieto fine della morte, vissuta (come il sesso) con disperata vitalità. (p.47)

La morte è dunque vista in modo ambivalente, come un estremo atto di vita; di più, l'atto di morte nei piani di Cris si intreccia all'atto del dare la vita concretizzato nell'orgasmo. Si tratta in tutto e per tutto di una «gaia morte», per dirlo con Bachtin (1979a): anche in Rabelais la morte è sempre data «su un piano grottesco e scherzoso; essa si interseca con la serie del mangiare e del bere, con quella degli escrementi e con quella anatomica» poiché «è fatta della stessa pasta di cui è fatta la vita» (pp.341-342). Una premonizione di questa scena finale si trovava già nelle prime pagine del romanzo, quando il protagonista sogna di trovarsi al cospetto di un sedicente «tribunale del popolo» con il fallo eretto: 
così mi accorgo che sono tutti vestiti di rosso e che io ho il Grande Proletario grosso, penso "come faccio in una situazione tanto pericolosa a avere il Grande Proletario grosso?" [...] e penso anche "adesso va giù, è stato un momento d'eccitazione, il caldo" ma no, niente da fare, il Grande Proletario è lì, rigido impettito davanti al tribunale del popolo. (Vassalli, 2008: p.14)

Vi è qui sicuramente lo sbeffeggiamento della "giustizia proletaria" esercitata dai brigatisti, ma al netto della parodia si può individuare la vitalità carnevalesca del corporeo che si contrappone alla condanna di morte. L'esibizione di un'esasperata vitalità nel momento del tribunale significa, in Vassalli, sottrarsi al giudizio di qualsiasi Chiesa: è quel disconoscimento della paura della morte tipico dello spirito del Gargantua evidenziato da Auerbach (1972). Per il critico tedesco,

in Rabelais non esiste peccato originale e nemmeno giudizio finale, e dunque non esiste una paura metafisica della morte. Come parte della natura, l'uomo gode della vita, delle funzioni del suo corpo e delle forze del suo spirito, e come tutte la altre creature della natura è soggetto alla dissoluzione naturale. (p.19) 
È difatti solo tramite questa ostentazione del corpo materiale e osceno che nel momento del suicidio Cris può liberarsi dalla pesantezza granitica delle ideologie con le loro parole chiave e, finalmente, «abitare il vento»:

allora il Grande Proletario comincerà a crescere e crescere, e si ergerà caldamente contro al bíschero universale cioè al mondo. [...] Poi io mi butto e nell'attimo proprio che la corda si tende al Grande Proletario gli si sbloccano le corde vocali, e canta. [...] L'orgasmo viene nell'attimo che si crepa e crepando si espelle la cosiddetta anima, io me lo spiego così. [...] Buttare l'anima in un orgasmo. Nel vento. (Vassalli, 2008: p.101)

\section{Conclusioni: trauma e strategie di "derealizzazione"}

Al termine di questa rassegna dei motivi del repertorio del carnevale nel romanzo di Vassalli, una volta compresa la qualità carnevalesca dell'elemento comico ed individuata la funzione contestativa di questa comicità, possiamo chiederci quale sia un più ampio senso complessivo, un senso per così dire culturale, di questa operazione a tutta prima comica. Per rispondere a questa domanda occorre delineare le coordinate dell' "habitat culturale" all'interno del quale il romanzo è stato composto. 
L'orizzonte immediato dell'autore è senza dubbio, come abbiamo dimostrato, la demitizzazione del linguaggio rivoluzionario, dinamitardo e "gruppettaro": un linguaggio fortemente connotato politicamente cui attingeva anche una parte dei componenti del Gruppo 63 e che aveva dato luogo a contrasti all'interno dello stesso gruppo. Pensiamo, per esempio, ai dibattiti ospitati su una delle riviste della neoavanguardia su cui pubblicò anche Vassalli: Quindici (Balestrini, 2008), uscita tra il 1967 e il 1969 e diretta sin dal numero 15 da Nanni Balestrini, uno degli autori più engagés del gruppo. La crescente politicizzazione della rivista provocò in effetti difficoltose spaccature all'interno della redazione stessa dal momento in cui si moltiplicarono, tra le sue pagine, le pubblicazioni dei documenti ufficiali dei movimenti studenteschi italiano, francese e tedesco, documenti intrisi di quel linguaggio che Vassalli tende a ridicolizzare in Abitare il vento. Vediamo dunque come la parodia, voltandosi verso il passato, si faccia in un certo modo portavoce di un compiuto dissenso interno a un gruppo letterario: una contestazione sorta nel momento in cui questo gruppo veniva a sovrapporsi e coincidere con le istanze dei movimenti politici del Sessantotto. Per questo motivo, come abbiamo accennato, si può sostenere che l'opera sia in parte frutto della delusione della pur breve esperienza neoavanguardista dell'autore.

Ma, se anche abbiamo posto l'accento sulla svuotamento di significato delle espressioni ricorrenti nel regime discorsivo della "Rivoluzione", il magmatico linguaggio comico del 
romanzo non si limita a queste formule, e identifica altri bersagli oggetto del "discorso impavido", assemblando materiali (che abbiamo definito "mitologemi") provenienti da altri campi semantici. Il protagonista mostra infatti di essere intriso di una cultura scolastica che viene disseminata nel testo mediante la citazione continua di espressioni desunte dalla più canonica storia della letteratura e persino dalla quarta di copertina di libri studiati a scuola: una locuzione che, completamente svuotata del suo significato e ridotta a puro tic linguistico, fa capolino nei torrenti verbali di Cris è «lirici greci nel'immortale risuono del verso quasimodiano» (ad esempio in Vassalli, 2008: p.17). Persino la formula del «Grande Proletario», che abbiamo assunto a cardine della carnevalizzazione demistificatrice del romanzo, è in effetti ambivalente. Non dobbiamo dimenticare infatti che il referente immediato dell'espressione è il celebre discorso pronunziato da Giovanni Pascoli nel 1911, all'alba dell'invasione italiana ai danni della Libia, discorso che verrà poi riportato in tutte le antologie di storia della letteratura italiana. Il riferimento è evidente nel primo utilizzo della locuzione da parte di Cris, in una citazione quasi integrale dell'incipit pascoliano: «il Grande Proletario si è mosso». Così la frase fatta non è solo un mitologema prodotto dalla macchina mitologica della "Rivoluzione", ma appartiene anche ad un altro regime discorsivo attorniato da un'aura sacrale e percepito pertanto come discorso dominante: il 
linguaggio della letteratura, nella sua declinazione più superficialmente scolastica e banalizzata.

Ma la commistione debordante di questi linguaggi stereotipati (a cui si possono aggiungere i giochi enigmistici svolti da Cris e integrati nel suo flusso di coscienza) non svolge solo la funzione demistificatrice che abbiamo fin qui descritto. Se infatti l'affrancamento dalle ideologie possiede una carica per così dire illuministica, tesa alla demitizzazione, Susanne Kleinert ha notato come in questa aspirazione non manchi anche una componente traumatica propria di chi ha vissuto un periodo che la memoria pubblica, con una forte connotazione negativa, ha etichettato come "anni di piombo". Il ridicolo e il comico carnevalesco sarebbero pertanto da comprendere in una più ampia strategia di "derealizzazione" in cui troviamo inclusi anche il linguaggio letterario, la componente ludica e quella metafinzionale. Kleinert (2006) ha individuato infatti, quale cifra del romanzo di Vassalli, un «humourisme linguistique qui cherche à dissoudre tous les postulats idéologiques dans un rabaissement carnavalesque du discours, au sens bakhtinien du terme», e non ha mancato di notare come «ce caractère traumatisant est aussi distancié par le jeu métafictionnel» (pp.353-354): la stessa qualità metafinzionale che contribuisce, come abbiamo visto in precedenza, a differenziare la comicità carnevalesca di Vassalli 
dalla satira pura mediante il coinvolgimento dell'autore stesso, chiamato in causa dal proprio personaggio.

Il linguaggio letterario, il discorso rivoluzionario e la dimensione ludica e metafinzionale vengono a comporre dunque un diaframma che l'autore pone fra sé e l'esperienza traumatizzante degli anni di piombo, in un'ottica di "derealizzazione" e distanziamento post-traumatici che Kleinert (2006) ha ben descritto:

le niveau de référence politique se voit en quelque sorte déréalisé par le langage du protagoniste, un langage fortement esthétisé, empreint d'une part de vulgarité, mais d'autre part d'une forme très litterérarisée: rimes fréquentes, jeaux de mots, ambiguïtés, allusions nombreuses à Quasimodo, éléments métafictionnels pirandelliens. Tout cela donne au discours du narrateur un caratère fortement ludique [...] le langage particulier de Abitare il vento revête aussi la fonction de distanciation face à une sombre réalité [...]. (pp.353-354)

Significativo, in questo senso, è l'anno di uscita del libro: quel 1980 che, sotto le macerie della strage alla stazione di Bologna (evento traumatico per eccellenza), seppellirà anche le grandi contestazioni e la lotta armata, dando il via all'epoca del cosiddetto "riflusso". Si tratta di un percorso collettivo che si rispecchia nella singolare parabola 
artistica dell'autore; scriverà infatti Vassalli in una postfazione all'edizione del 2008 di Abitare il vento, intitolata Trent'anni dopo. Riflessioni su un personaggio e la sua storia:

Antonio Cristiano Rigotti, come Jacopo Ortis, era un personaggio senza sbocchi. [...] Ma era anche una presenza imprescindibile, in quegli anni, nella cultura e nella società del nostro Paese: e io dovevo liberarmene per poter arrivare a una scrittura più distesa e per potermi occupare di altri personaggi, forse altrettanto tragici ma più interessanti. Dino Campana, la strega di Zardino, Mattio Lovat... (Vassalli, 2008: p.110)

Una simile liberazione da un'eredità traumatica è perseguita dal protagonista del romanzo di Roth, che già dal titolo riprende in forma parodica il libro biblico delle Lamentazioni in cui si piange la distruzione di Gerusalemme (il riferimento biblico nel titolo è un'altra coincidenza tra i due testi). Attribuendo alla sua ossessione sessuale la qualità di sintomo, Alex Portnoy, nel suo ostentato e ambivalente sbeffeggiamento della lingua ebraica, esprime il senso patologico di un trauma che non ha nemmeno esperito direttamente, ma ricevuto in eredità familiare e culturale:

perché, solo nel mio letto di New York, perché continuo a menarmelo senza remissione? Dottore, come si chiama questa mia malattia? È la pena ebraica di cui ho sentito tanto 
parlare? È l'eredità trasmessami dai pogrom e dalle persecuzioni? Dallo scherno e dagli insulti distribuiti dai goyim negli ultimi duemila piacevoli anni? (Roth, 2014: p.32)

Così, anche quella del protagonista di Roth è una reazione esasperatamente sessualizzata e carnevalesca, che sfocia nella dimensione dissacrante al momento della ribellione filiale a questa eredità: «fammi il piacere, popol mio: prendi la tua sofferente eredità e ficcatela su per il tuo sofferente culo...si dà anche il caso che io sia un essere umano!» (Roth, 2014: p.62). Se dunque una relazione tra i due romanzi andrebbe esplorata più approfonditamente, non possiamo evitare di mettere in risalto i punti di contatto, patenti in particolare nella quête sessuale e nell'abbassamento di un linguaggio percepito come sacrale per esorcizzare un trauma.

Per quanto riguarda Abitare il vento, infine, con quest'opera Sebastiano Vassalli chiude dunque decisamente il suo personale capitolo della neoavanguardia, dello sperimentalismo e della contestazione politica. Di più, prendendo di mira le parole d'ordine di quella stagione, intraprende un vero e proprio processo di decostruzione dell'immagine mitica del Sessantotto così come viene tramandata ai pur sparuti movimenti antagonisti sopravvissuti a partire dagli anni Ottanta. Ma non bisogna sottovalutare l'esperienza traumatica che, forse ancora di più della tensione illuministica che anima la ricerca di Vassalli sul linguaggio, è il 
vero motore di questo processo comico; di una comicità che, in ultima analisi, assume tratti analoghi a quelli di un'ansia di rimozione.

\section{Reference list}

Auerbach E (1972) Mimesis (II). Torino: Einaudi.

Bachtin M (1979a) Estetica e romanzo. Torino: Einaudi.

Bachtin M (1979b) L'opera di Rabelais e la cultura popolare. Riso, carnevale e festa nella tradizione medievale e rinascimentale. Torino: Einaudi.

Balestrini N ed. (2008) Quindici. Una rivista e il Sessantotto. Milano: Feltrinelli.

Cicala R (2011) La sperimentazione editoriale del giovane Vassalli (con bibliografia 19651984, catalogo delle sue edizioni CDE e Ant.Ed, immagini e testi). Novara: Interlinea.

Corti M (1978) Il viaggio testuale. Torino: Einaudi.

Ioli G (1982) La satira e il "riso" nel romanzo di Sebastiano Vassalli. Prometeo 2 (2): 4757

Jesi F (2011) Cultura di destra. Roma: Nottetempo. 
Kleinert S (2006) Violence politique et sentiment d'irrealité: la représentation des années ' 70 chez Balestrini, Camon et Vassalli. In: Actes de la Conférence Internationale: La valeur de la letterature pendant et après les anées '70 - le cas de l'Italie et du Portugal. (eds. M Jansen and P Jordão), Utrecht, NL, 11-13 March 2004, p.336-356. Utrecht: Italianistica Ultraiectina 1.

Nesi C (2005) Sebastiano Vassalli. Fiesole: Cadmo.

Rabelais F (1973) Gargantua e Pantagruele. Torino: Einaudi.

Rabelais F (2012) Gargantua e Pantagruele, Roma: Newton Compton.

Roth P (2014) Lamento di Portnoy, Torino: Einaudi.

Vassalli S (1976) L'arrivo della lozione. Torino: Einaudi.

Vassalli S (1982) Mareblù. Milano: Arnoldo Mondadori.

Vassalli S (2008) Abitare il vento. Milano: Calypso. 
Vassalli S and Tesio G (2010) Un nulla pieno di storie. Ricordi e considerazioni di un viaggiatore nel tempo. Novara: Interlinea. 